\title{
Search for Single Top (FCNC) and Heavy and Excited Fermions at LEP
}

\section{Mário Pimenta* LIP/IST, Lisboa, Portugal}

E-mail: pamenta@īip.pti

Abstract: Recent results on searches for flavour changing neutral currents in the Top sector and for heavy and excited leptons at LEP are reported.

Searches for different possible extensions of the fermion sector of the Standard Model (SM) are summarised in this report. The detailed descriptions of the analyses can be found in several contributions to this conference made by the four LEP collaborations ,ALEPH [i] data were collected at centre-of-mass energies ranging from $189 \mathrm{GeV}$ to $208 \mathrm{GeV}$ and with luminosities between $350 \mathrm{pb}^{-1}$ and $650 \mathrm{pb}^{-1}$. All results are preliminary and all limits set are at $95 \%$ Confidence Level (CL).

\section{Search for Heavy and Excited Leptons}

\begin{tabular}{|cccc|}
\hline Decay Mode & Model & Dirac & Majorana \\
\hline \multirow{3}{*}{$L^{0} \rightarrow e W$} & Sequential & 101.3 & 89.5 \\
& Vector & 102.6 & - \\
& Mirror & 100.8 & 89.5 \\
\hline \multirow{3}{*}{$L^{0} \rightarrow \mu W$} & Sequential & 101.5 & 90.7 \\
& Vector & 102.7 & - \\
& Mirror & 101.0 & 90.7 \\
\hline \multirow{2}{*}{$L^{0} \rightarrow \tau W$} & Sequential & 90.3 & 80.5 \\
& Vector & 99.3 & - \\
& Mirror & 90.3 & 80.5 \\
\hline \hline
\end{tabular}

Table 1: L3 lower masses limits in $\mathrm{GeV} / c^{2}$ for pairproduced neutral heavy leptons.

case of excited leptons the $\mathrm{SU}(2)$ and $\mathrm{U}(1)$ couplings are give
$f$ and $f^{\prime}$ respectively, and several scenarios are possible.
New leptons couple to gauge bosons through their $\mathrm{SU}(2) \times \mathrm{U}(1)$ charges and can be be pair produced at LEP. The pair production cross section is proportional to the pair production cross section of the corresponding SM fermions, but affected by a factor $g$ which depends on the type of the lepton (for instances $\mathrm{g}=2$ for vector leptons) and by a space phase factor which is equal to zero at the kinematic limit.

Single production of new leptons is also possible at LEP through their mixing with the SM leptons. In the

\footnotetext{
${ }^{*}$ Speaker.
} 
New leptons can decay into SM leptons by radiating a $\gamma, Z$ or $W$, according to the type of lepton and the scenario considered. In the case of the charged heavy lepton the possibilities of decaying into its neutral partner or even not decaying inside the detector were also considered.

The combination of the several production and decay modes gives rises to many and very different topologies, from final states with only $\gamma \mathrm{s}$ to isolated leptons or jets. In most of the topologies there is an important missing energy. The main backgrounds are $W W, q \bar{q}(\gamma)$ and $Z Z$.

No signal for new heavy or excited leptons was observed. The lower limits on the masses of heavy leptons obtained by L3 on the pair production of neutral and charged leptons are shown in Tables and 2 respectively. The lower limits on the masses of pair produced excited leptons obtained by DELPHI and OPAL are shown in Table B. $_{i}^{-}$Two scenarios were considered: $f=f^{\prime}$ and $\bar{f}=-f^{\prime}$.

\begin{tabular}{|ccc|}
\hline Decay Mode & Model \\
\hline \multirow{3}{*}{$L^{ \pm} \rightarrow \nu W$} & Sequential & 100.8 \\
& Vector & 101.2 \\
& Mirror & 100.5 \\
\hline \multirow{3}{*}{$L^{ \pm} \rightarrow L^{0} W$} & Sequential & 101.9 \\
& Vector & 102.1 \\
& Mirror & 101.9 \\
\hline \multirow{3}{*}{ STABLE } & Sequential & 102.6 \\
& Vector & 102.6 \\
& Mirror & 102.6 \\
\hline
\end{tabular}

Table 2: L3 lower masses limits in $\mathrm{GeV} / c^{2}$ for pair-produced charged heavy leptons.

\begin{tabular}{|ccccccc|}
\hline & $e^{*}$ & $\mu^{*}$ & $\tau^{*}$ & $\nu_{e}^{*}$ & $\nu_{\mu}^{*}$ & $\nu_{\tau}^{*}$ \\
\hline \hline$f=f^{\prime}$ & 103.0 & 103.1 & 102.2 & 102.0 & 102.4 & 95.3 \\
$f=-f^{\prime}$ & 98.0 & 98.0 & 98.0 & 102.7 & 102.8 & 102.8 \\
\hline \hline$f=f^{\prime}$ & 102.9 & 102.9 & 102.8 & - & - & - \\
\hline
\end{tabular}

Table 3: Lower limits (in $\mathrm{GeV} / \mathrm{c}^{2}$ ) at $95 \% \mathrm{CL}$ on the excited lepton masses from the pair production modes. The first two lines corresponds to results obtained by DELPHI while the last line reports those obtained by OPAl.

For the single production of excited leptons DELPHI and OPAL derived limits on the ratio of the coupling $(f)$ to the Compositness scale $(\Lambda)$ as a function of the excited lepton mass. In the case of charged excited leptons (electrons, muons and taus) the result obtained by the LEP Exotica Working Group combining the DELPHI and OPAL results (with $f=f^{\prime}$ ) are shown in figure $i_{-i}^{-}$The stronger limit in the case of electrons is due to the contribution of the t-channel diagram.

The limit on the excited electron production for $f=f^{\prime}$ can be extended above the LEP kinematic threshold searching for deviations in the $e^{+} e^{-} \rightarrow \gamma \gamma(\gamma)$ differential cross

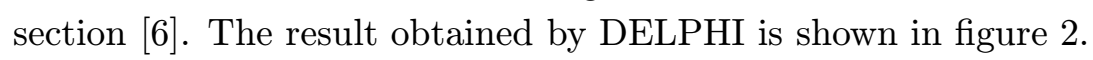

For the single production of isosinglet heavy neutrinos L3 extended the limits on the mixing angle to a mass range above the $W$ mass as is shown in figure

\section{Search for Single Top production}

The most prominent signature for the direct observation of Flavour Changing Neutral Currents (FCNC) processes is the single Top quark production in the reaction $e^{+} e^{-} \rightarrow t \bar{c}$ 
(or $e^{+} e^{-} \rightarrow t \bar{c}$ ). At tree level the single production of Top can be introduced in the $s$-channel diagram by two anomalous couplings $K_{z}$ and $K_{\gamma}$ associated to the exchange of a $Z$ and a $\gamma$, respectively. A more general scenario is to consider four fermion interactions with the possibility of scalar, vector and tensor couplings.

No significant excess was observed and limits in the the plane $\left(k_{z}, K_{\gamma}\right)$ were set as shown in figure The light grey region show the LEP exclusion region for $m_{t}=174 \mathrm{GeV} / \mathrm{c}^{2}$ with QCD and ISR corrections. The exclusion curves for different values of Top quark masses are also shown. The hatched area shows the CDF exclusion region $[\overline{\bar{i}} \mathbf{1}]]$ and the arrows shows the ZEUS $K_{t u_{\gamma}}$ exclusion [i i explored by DELPHI in the general framework of four-fermion contact interactions. The limits on the energy scale $\Lambda$ are shown in table

\section{Acknowledgments}

Warm thanks are due to the extraordinary achievements of the LEP accelerator team, reaching centre-of-mass energies well above the specifications. I am in debt to the four LEP collaborations for making available the most recent results and in particular to the LEP Exotica Working Group and the LIP DELPHI team for their help and comments during the preparation of this talk.

\begin{tabular}{|cc|}
\hline $\begin{array}{c}\text { Scenario } \\
(\mathrm{i}, \mathrm{j}=\mathrm{L}, \mathrm{R})\end{array}$ & $\begin{array}{c}\text { Limit in } \\
\Lambda(\mathrm{GeV})\end{array}$ \\
\hline$T_{R R}=S_{R R}=V_{i j}=1 ; a_{j}^{Z}=0$ & 1312 \\
\hline$T_{R R}=1 ; S_{R R}=V_{i j}=a_{j}^{Z}=0$ & 1143 \\
\hline$S_{R R}=1 ; T_{R R}=V_{i j}=a_{j}^{Z}=0$ & 604 \\
\hline$T_{R R}=S_{R R}=0 ; V_{i j}=1 ; a_{j}^{Z}=0$ & 986 \\
\hline$T_{R R}=S_{R R}=V_{i j}=0 ; a_{j}^{Z}=1$ & 473 \\
\hline$T_{R R}=S_{R R}=0 ; V_{i j}=a_{j}^{Z}=1$ & 992 \\
\hline$T_{R R}=S_{R R}=0 ; V_{i j}=1 ; a_{j}^{Z}=-1$ & 1003 \\
\hline
\end{tabular}

Table 4: Observed and expected limits $\Lambda$ obtained by DELPHI exploring several four-fermion contact interactions scenarios

\section{References}

[1] ALEPH Coll., M. Antonelli, F. Cerutti, in Proceedings of the EPS International Conference on High Energy Physics, Budapest, 2001 (D. Horvath, P. Levai, A. Patkos, eds.), JHEP (http://jhep.sissa.it/) Proceedings Section, PrHEP-hep2001/248.

[2] DELPHI Coll., W.Adam et al., in Proceedings of the EPS International Conference on High Energy Physics, Budapest, 2001 (D. Horvath, P. Levai, A. Patkos, eds.), JHEP (http://jhep.sissa.it/) Proceedings Section, PrHEP-hep2001/320; DELPHI Coll., S.Andringa et al., in Proceedings of the EPS International Conference on High Energy Physics, Budapest, 2001 (D. Horvath, P. Levai, A. Patkos, eds.), JHEP (http://jhep.sissa.it/) Proceedings Section, PrHEP-hep2001/335; DELPHI Coll., S.Andringa et al., in Proceedings of the EPS International Conference on High Energy Physics, Budapest, 2001 (D. Horvath, P. Levai, A. Patkos, eds.), JHEP (http://jhep.sissa.it/) Proceedings Section, PrHEP-hep2001/336.

[3] L3 Coll., in Proceedings of the EPS International Conference on High Energy Physics, Budapest, 2001 (D. Horvath, P. Levai, A. Patkos, eds.), JHEP (http://jhep.sissa.it/) Proceedings Section, PrHEP-hep2001/516; 
L3 Coll., in Proceedings of the EPS International Conference on High Energy Physics, Budapest, 2001 (D. Horvath, P. Levai, A. Patkos, eds.), JHEP (http://jhep.sissa.it/) Proceedings Section, PrHEP-hep2001/517.

[4] OPAL Coll., in Proceedings of the EPS International Conference on High Energy Physics, Budapest, 2001 (D. Horvath, P. Levai, A. Patkos, eds.), JHEP (http://jhep.sissa.it/) Proceedings Section, PrHEP-hep2001/159.

[5] LEP Exotica Working Group, in Proceedings of the EPS International Conference on High Energy Physics, Budapest, 2001 (D. Horvath, P. Levai, A. Patkos, eds.), JHEP (http://jhep.sissa.it/) Proceedings Section, PrHEP-hep2001/281;

LEP Exotica Working Group, in Proceedings of the EPS International Conference on High Energy Physics, Budapest, 2001 (D. Horvath, P. Levai, A. Patkos, eds.), JHEP (http://jhep.sissa.it/) Proceedings Section, PrHEP-hep2001/282.

[6] DELPHI Collab., S.Andringa et al., in Proceedings of the EPS International Conference on High Energy Physics, Budapest, 2001 (D. Horvath, P. Levai, A. Patkos, eds.), JHEP (http://jhep.sissa.it/) Proceedings Section, PrHEP-hep2001/282.

[7] CDF Collab., F.Abe et al., Phys. Rev. Lett. 80 (1998) 2525.

[8] ZEUS Collab., in Proceedings of the EPS International Conference on High Energy Physics, Budapest, 2001 (D. Horvath, P. Levai, A. Patkos, eds.), JHEP (http://jhep.sissa.it/) Proceedings Section, PrHEP-hep2001/650.

[9] H1 Collab., in Proceedings of the EPS International Conference on High Energy Physics, Budapest, 2001 (D. Horvath, P. Levai, A. Patkos, eds.), JHEP (http://jhep.sissa.it/) Proceedings Section, PrHEP-hep2001/789. 


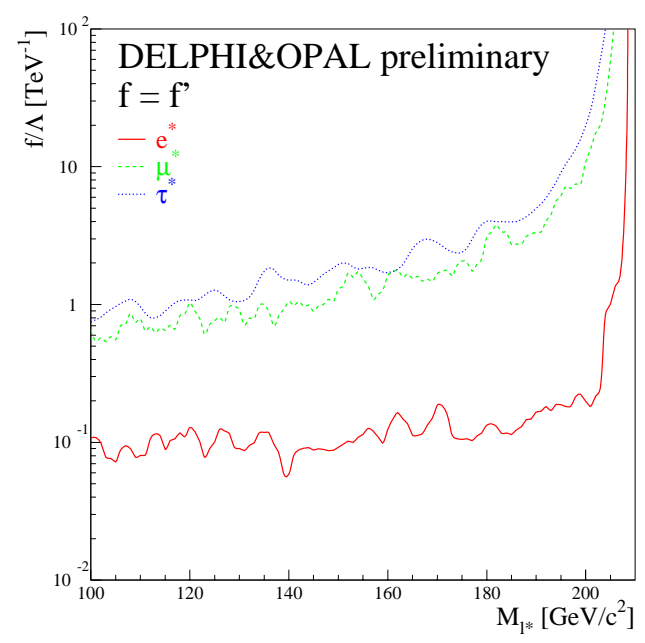

Figure 1: upper limits on the ratio of the coupling to the compositness scale from the search for single excited leptons. The lines correspond to production of excited electrons (solid), muons (dashed) and taus (dotted)

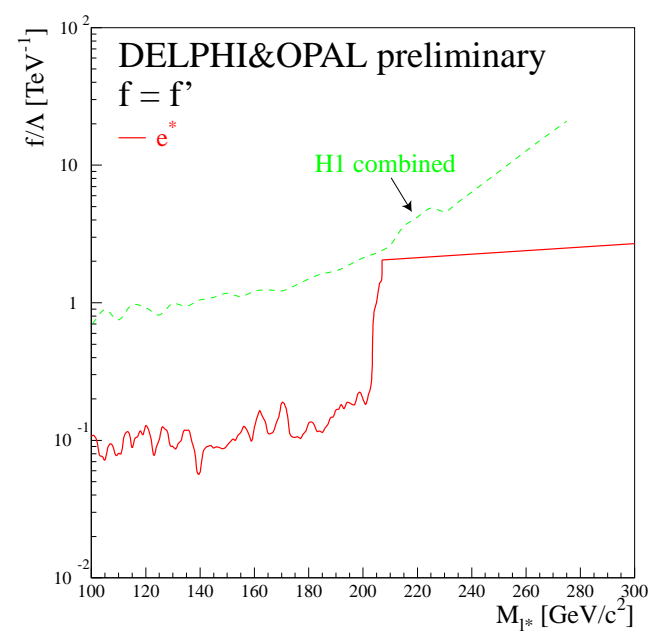

Figure 2: DELPHI combined limit on excited electron production for $f=f^{\prime}$ from direct and indirect searches. 


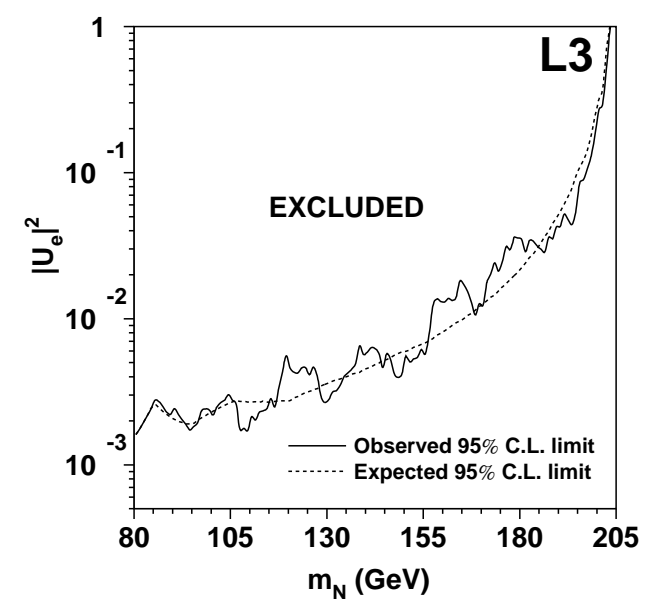

Figure 3: L3 observed and expected upper limits on the mixing amplitude $|U-e|^{2}$ as a function of the heavy isosinglet mass $m_{N}$

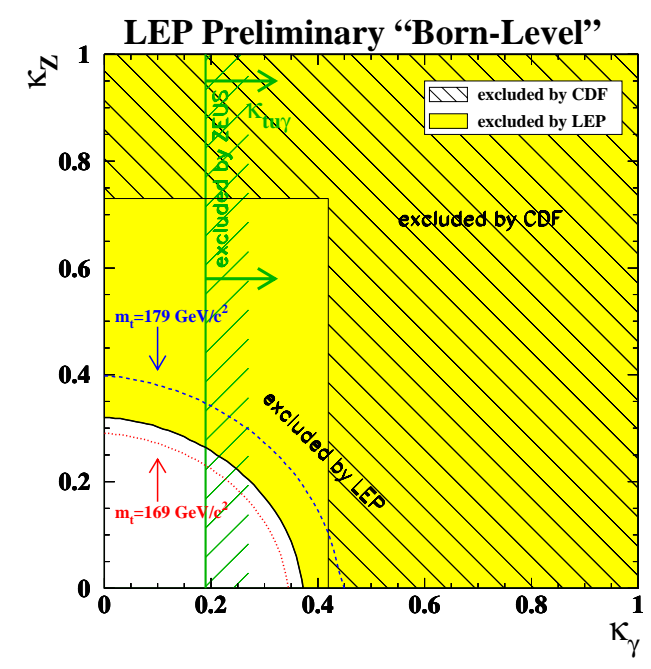

Figure 4: Combined limits in the $\left(k_{z}, K_{\gamma}\right)$ plane. 